\title{
FINE UNIFORMITY AND THE LOCALLY FINITE HYPERSPACE TOPOLOGY
}

\author{
S. A. NAIMPALLY AND P. L. SHARMA \\ (Communicated by Doug W. Curtis)
}

\begin{abstract}
It is shown that a uniformizable space $X$ is normal iff the locally finite topology $e^{\tau}$ on the hyperspace $2^{X}$ coincides with the topology transmitted by the fine uniformity of $X$. We also prove that, for $X$ normal, the topology $e^{\tau}$ is first countable only if the set of limit points $X^{\prime}$ of $X$ is countably compact. Applications of these results to pseudocompactness and Atsuji spaces are given.
\end{abstract}

1. Introduction. In a recent paper [4], Beer et al. proved that the locally finite topology $e^{\tau}$ on the hyperspace $2^{X}$ of a metrizable space $X$ is the supremum of all the Hausdorff metric topologies corresponding to equivalent metrics on $X$. In view of this result, and because a Hausdorff metric topology generally lacks topological invariance, the authors of [4] justifiably contended that the locally finite topology on a hyperspace would be a more useful structure in most applications. In this paper, we determine the appropriate context for the locally finite topology, namely that this topology is a uniform notion. Specifically, we prove that, in the class of uniformizable spaces, it is precisely the normal spaces for which the locally finite topology on a hyperspace coincides with the topology transmitted by the fine uniformity of the space. This result specialized to metrizable spaces yields the aforementioned result of Beer et al. We also show that if $X$ is paracompact, then for $e^{\tau}$ to be first countable, $X^{\prime}$ must be compact. From this, several known characterizations of Atsuji spaces follow.

All topological spaces considered in this paper are assumed to be $T_{1}$. Much of our terminology about hyperspaces is the same as in Michael's paper [11].

Let $(X, \tau)$ be a $T_{1}$ space. The hyperspace $2^{X}$ of $X$ is the set $\{E \subseteq X: E$ is closed, $E \neq \varnothing\}$. For a collection $\mathscr{A}$ of subsets of $X$, we write

$$
\begin{aligned}
& \mathscr{A}^{-}=\left\{E \in 2^{X}: E \cap A \neq \varnothing \forall A \in \mathscr{A}\right\}, \\
& \mathscr{A}^{+}=\left\{E \in 2^{X}: E \subseteq \bigcup \mathscr{A}\right\}, \\
& \mathscr{A}^{-}=\mathscr{A}^{-} \cap \mathscr{A}^{+} .
\end{aligned}
$$

Received by the editors September 16, 1986 and, in revised form, March 9, 1987.

1980 Mathematics Subject Classification (1985 Revision). Primary 54B20, 54A10; Secondary 54D30, 54E15.

Key words and phrases. Hyperspace, locally finite topology, fine uniformity, Hausdorff metric, pseudocompactness.

The second author's research was partially supported by a visiting professorship (July 1986) through an NSERC (CANADA) grant of the first author, and by a Butler University Faculty Fellowship. 
The finite (locally finite) topology $2^{\tau}\left(e^{\tau}\right)$ on $2^{X}$ is the one generated by the sets

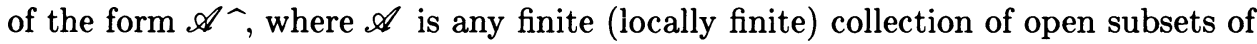
$X$.

It is easily seen that all collections of the form $\mathscr{A}^{-}$and $\{A\}^{+}$, where $A$ is open in $X$, and $\mathscr{A}$ is a finite (locally finite) collection of open subsets of $X$, is a subbase for $2^{\tau}\left(e^{\tau}\right)$. Whenever $A$ is a subset of $X$, it is convenient to write $A^{+}$for $\{A\}^{+}$, and from now on, we will do so. The following result, analogous to Lemma 2.3 of [11], can be easily verified.

LEMMA 1.1. Let $\mathscr{A}$ and $\mathscr{B}$ be two locally finite collections of nonempty, open subsets of a $T_{1}$ space $X$. Then:

(a) $\mathscr{A}^{\wedge} \subseteq \mathscr{B}^{\wedge}$ iff each member of $\mathscr{B}$ contains a member of $\mathscr{A}$ and $\bigcup \mathscr{A} \subseteq \bigcup \mathscr{B}$.

(b) If $E \subseteq X$ is such that $E \subseteq \cup \mathscr{A}$ and $E \cap A$ is finite and nonempty for all $A \in \mathscr{A}$, then $E \in 2^{X}, E$ is discrete in $X$, and $E \in \mathscr{A}^{-}$.

If $\mathscr{U}$ is a uniformity on a set $X,|\mathscr{U}|$ denotes the topology on $X$ induced by $\mathscr{U}$. For a uniformizable topological space $(X, \tau)$, the finest compatible uniformity on $X$, called the fine uniformity for $\tau$, will be denoted by $\mathscr{U}(\tau)$. Similarly, the finest compatible totally bounded uniformity will be denoted by $\mathscr{U}^{*}(\tau)$. If $d$ is a metric on a set $X, \mathscr{U}(d)$ (resp. $\tau(d)$ ) denotes the uniformity (resp. topology) induced by $d$ on $X$.

Let $(X, \tau)$ be a uniformizable topological space, and let $\mathscr{U}$ be a compatible uniformity on $X$. For each $U \in \mathscr{U}$, let $\mathrm{U}=\left\{(A, B): A, B \in 2^{X}, A \subseteq U(B)\right.$ and $B \subseteq U(A)\}$. Then, $\{\mathbf{U}: U \in \mathscr{U}\}$ is a base for a uniformity $2^{\mathscr{U}}$ on $2^{X}$. If $\mathscr{U}=\mathscr{U}(d)$ for some metric $d$ on $X$, the uniformity $2^{\mathscr{U}}$ on $2^{X}$ is simply the uniformity of the Hausdorff metric determined by $d$ (see Willard [15, p. 250]).

A $T_{1}$ space $X$ is said to be feebly compact provided each locally finite family of open subsets of $X$ is finite. Clearly, every countably compact space is feebly compact, and any feebly compact space is necessarily pseudocompact. Conversely, a completely regular, pseudocompact space is feebly compact [5], and for normal spaces, the three concepts coincide.

It is obvious that the collection $F(X)$, of all finite subsets of a $T_{1}$ space $X$ is always dense in $\left(2^{X}, 2^{\tau}\right)$. However, if $X$ contains an infinite, locally finite collection of open sets, $F(X)$ is not dense in $\left(2^{X}, e^{\tau}\right)$. This leads us to the following result.

THEOREM 1.2. A $T_{1}$ space $(X, \tau)$ is feebly compact iff $2^{\tau}=e^{\tau}$.

Whenever $2^{X}$ is given either of the topologies $2^{\tau}$ or $e^{\tau}$, the function $x \rightarrow\{x\}$ embeds $X$ as a closed subspace of $2^{X}$. Thus, if some hereditary topological property does not hold in $X$, it will also not hold in $2^{X}$.

THEOREM 1.3. The following conditions are equivalent:

(a) $(X, \tau)$ is regular.

(b) $\left(2^{X}, 2^{\tau}\right)$ is Hausdorff.

(c) $\left(2^{X}, e^{\tau}\right)$ is Hausdorff.

PROOF. The proof of $(a) \Leftrightarrow(b)$ is given in Michael's paper [11]; and (b) $\Rightarrow(c)$ because $2^{\tau} \subseteq e^{\tau}$. The proof of $(\mathrm{c}) \Rightarrow(\mathrm{a})$ is similar to Michael's proof of (b) $\Rightarrow(\mathrm{a})$, and we will omit it. 
2. Main results. In this section, we will show that if $(X, \tau)$ is uniformizable, then $X$ is normal iff $e^{\tau}=\left|2^{\mathscr{U}(\tau)}\right|$. We also show that $X^{\prime}$ is countably compact if $\left(2^{X}, e^{\tau}\right)$ is first countable and $X$ normal.

THEOREM 2.1. Let $(X, \tau)$ be a uniformizable space, and let $\mathscr{U}$ be any compatible uniformity on $X$. Then:

(a) $\left|2^{\mathscr{U}}\right| \subseteq e^{\tau}$.

(b) $\left|2^{\mathscr{U}}\right| \subseteq 2^{\tau} \Leftrightarrow \mathscr{U}$ is totally bounded.

Proof. (a) Take any $A \in 2^{X}$, and any symmetric $U \in \mathscr{U}$. It will suffice to show that there is a $\gamma \in e^{\tau}$, such that $A \in \gamma \subseteq \mathbf{U}(A)$. Choose a symmetric open $V \in \mathscr{U}$ such that $V^{5} \subseteq U$, and let $E \subseteq A$ be maximal such that for any $x, y \in E$, $x \neq y \Rightarrow(x, y) \notin V^{4}$. Note that for each $x \in X, V(x)$ intersects at most one member of the collection $\mathscr{A}=\{V(t): t \in E\} ; \mathscr{A}$ is a discrete collection of open subsets of $X$, and $A \in \gamma=\mathscr{A}^{-} \cap V(A)^{+}$. We claim that $\gamma \subseteq \mathbf{U}(A)$. Clearly, if $B \in \gamma$ then $B \subseteq V(A) \subseteq U(A)$. Also, for any $x \in A$, we can, by the maximality condition on $E$, find an $s \in E$ such that $(x, s) \in V^{4}$. As $B \in \mathscr{A}^{-}, B \cap V(s) \neq \varnothing$; and if $y \in B \cap V(s),(x, y) \in V^{5}$. Thus $x \in V^{5}(y) \subseteq U(B)$. This shows that $A \subseteq U(B)$. As we already have $B \subseteq U(A),(A, B) \in \mathbf{U}$. Thus, $\gamma \subseteq \mathbf{U}(A)$, and the proof is complete.

(b) See Lemma 3.2 of Michael's paper [11].

THEOREM 2.2. Let $(X, \tau)$ be normal. Then:

(a) $\left|2^{\mathscr{U}(\tau)}\right|=e^{\tau}$.

(b) $\left|2^{\mathscr{U} *^{*}(\tau)}\right|=2^{\tau}$.

ProOF. (a) Take $A \in 2^{X}$, an arbitrary open set $G$ of $X$, and an arbitrary, locally finite collection $\mathscr{A}=\left\{G_{i}: i \in I\right\}$ of open subsets of $X$, such that $A \in \mathscr{A}^{-} \cap G^{+}$. Since $X$ is normal, there exists a continuous map $\theta: X \rightarrow[0,1]$ such that $\theta(A)=0$, and $\theta(X-G)=1$. The pseudometric $\theta^{*}$ on $X$ defined by $\theta^{*}(x, y)=|\theta(x)-\theta(y)|$, is continuous, and thus, uniform with regard to the uniformity $\mathscr{U}(\tau)$ on $X$ (see [5]). For $W=\left\{(x, y): \theta^{*}(x, y)<1\right\}, W(A) \subseteq G$, and so, $\mathbf{W}(A) \subseteq G^{+}$. Now, for each $i \in I$, pick an $x_{i} \in A \cap G_{i}$, and let $E=\left\{x_{i}: i \in I\right\}$. Again, for each $i \in I$, let $g_{i}: X \rightarrow[0,1]$ be continuous such that $g_{i}\left(x_{i}\right)=0$, and $g_{i}\left(X-G_{i}\right)=1$. Then, the local finiteness of the collection $\mathscr{A}$ ensures that the function $d(x, y)=$ $\sum\left|g_{i}(x)-g_{i}(y)\right|, i \in I$, is a well-defined, continuous pseudometric on $X$, and thus, like $\theta^{*}$, it too is uniform with regard to $\mathscr{U}(\tau)$. We claim that for $V=$ $\{(x, y): d(x, y)<1 / 2\}, \mathbf{V}(A) \subseteq \mathscr{A}^{-}$. We first note that $V\left(x_{i}\right) \subseteq G_{i}$ for each $i \in I$. Now, take any $B \in \mathbf{V}(A)$, and any $i \in I$. Then, $A \subseteq V(B)$, and so, there exists some $b \in B$, such that $x_{i} \in V(b)$. Consequently, $b \in V\left(x_{i}\right) \subseteq G_{i}, B \cap G_{i} \neq \varnothing$. It follows that $B \in \mathscr{A}^{-}$, and therefore, $\mathbf{V}(A) \subseteq \mathscr{A}^{-}$. Thus, we have shown that $\mathbf{V}(A) \cap \mathbf{W}(A) \subseteq \mathscr{A}^{-} \cap G^{+}$.

(b) See Michael's paper [11].

THEOREM 2.3. Let $(X, \tau)$ be completely regular. The following conditions are equivalent:

(a) $(X, \tau)$ is normal.

(b) $\left|2^{\mathscr{U}(\tau)}\right|=e^{\tau}$.

(c) $\left|2^{\mathscr{U ^ { * }}(\tau)}\right|=2^{\tau}$ 
Proof. By the preceding theorem, $(\mathrm{a}) \Rightarrow(\mathrm{b})$, and $(\mathrm{a}) \Rightarrow(\mathrm{c})$. We will show that if $(X, \tau)$ is not normal, then for any compatible uniformity $\mathscr{U}$ on $X,\left|2^{\mathscr{U}}\right|$ does not contain $2^{\tau}$. This will show that each of (b) and (c) implies (a). The proof of (c) $\Rightarrow(\mathrm{a})$ is also implicitly contained in Michael's paper [11].

Take $A, B \in 2^{X}, A$ and $B$ disjoint, such that each open subset of $X$ containing $A$ intersects each open subset of $X$ containing $B$. As $(X-B)^{+}$is a neighborhood of $A$ in $2^{X}$ with regard to both $2^{\tau}$ and $e^{\tau}$, it will suffice to show that no neighborhood of $A$ in $\left(2^{X},\left|2^{\mathscr{U}}\right|\right)$ is contained in $(X-B)^{+}$. Note that for each $U \in \mathscr{U}, U(A) \cap B$ is nonempty; for, if $U(A) \cap B$ were empty for some $U \in \mathscr{U}$, then by choosing a $V \in \mathscr{U}$ such that $V^{2} \subseteq U$, we would have $V(A) \cap V(B)=\varnothing$, contrary to the assumed condition on the pair $A, B$. Now, for any $U \in \mathscr{U}$ and $b \in U(A) \cap B$, if $A^{*}=A \cup\{b\}$, then $A^{*} \in \mathrm{U}(A)$, whereas $A^{*} \notin(X-B)^{+}$. This completes the proof.

Let $(X, \tau)$ be completely regular. The partial order between the topologies $e^{\tau}$, $2^{\tau},\left|2^{\mathscr{U}(\tau)}\right|$, and $\left|2^{\mathscr{U}^{*}(\tau)}\right|$, as implied by Theorem 2.1, is summarized in the following Hasse diagram:

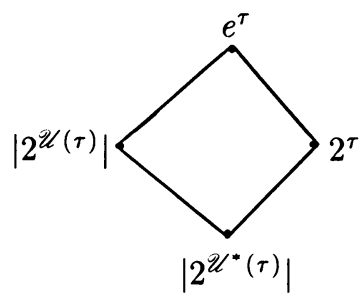

The four topologies considered in this diagram are all distinct iff the completely regular space $(X, \tau)$ is neither normal nor pseudocompact; all of the four topologies coincide iff $(X, \tau)$ is both normal and pseudocompact. We have $\left|2^{\mathscr{U} *^{*}(\tau)}\right|=\left|2^{\mathscr{U}(\tau)}\right| \subset$ $2^{\tau}=e^{\tau}$ iff $(X, \tau)$ pseudocompact but not normal, and $\left|2^{\mathscr{U}^{*}(\tau)}\right|=2^{\tau} \subset\left|2^{\mathscr{U}(\tau)}\right|=e^{\tau}$ iff $(X, \tau)$ is normal but not pseudocompact.

We recall that a metric $d$ on a set $X$ is called a UC metric (and then $(X, d)$ is called a UC space) provided each continuous, real-valued function on $(X, d)$ is uniformly continuous [14]. Equivalently, $(X, d)$ is a UC space iff $\mathscr{U}(d)$ is the fine uniformity of $(X, \tau(d))$. We call a topological space Atsuji provided $X$ is metrizable and the set of limit points $X^{\prime}$ of $X$ is compact. It is well known that a topological space $(X, \tau)$ admits a UC metric iff $(X, \tau)$ is an Atsuji space [13].

From the preceding theorem, we infer that if $(X, d)$ is a UC space then $\left|2^{\mathscr{U}(d)}\right|=$ $e^{\tau(d)}$. The converse of this also holds (cf. Theorems 12.18 and 15.1 of [12]), so that we have the following characterization of UC spaces given in [4].

COROllary 2.5. Let $(X, d)$ be a metric space. Then, $(X, d)$ is a UC space iff $\left|2^{\mathscr{U}(d)}\right|=e^{\tau(d)}$.

Let $(X, \tau)$ be a metrizable space, ard let $D_{0}$ be the set of all continuous pseudometrics on $X$. Also, let $D_{1}$ be the set of all metrics on $X$ which induce the topology $\tau$. Take a $d_{1} \in D_{1}$ and let $D_{2}=\left\{d \vee d_{1}: d \in D_{0}\right\}$. Clearly, $D_{2} \subseteq D_{1} \subseteq D_{0}$. It is obvious (cf. $\S 15.3$ of [6]) that the supremum of all the uniformities on $X$, one corresponding to each $d \in D_{2}$, is $\mathscr{U}(\tau)$. This statement also holds if $D_{2}$ is replaced by $D_{1}$ or $D_{0}$. Consequently, the various Hausdorff metric uniformities on $2^{X}$, one corresponding to each $d \in D_{1}$, together form a subbase for $2^{\mathscr{U}(\tau)}$. Thus, we have the following result, proved in [4]: 
THEOREM 2.6. Let $(X, \tau)$ be a metrizable space. Then, the supremum of all the Hausdorff metric topologies on $2^{X}$ corresponding to equivalent metrics on $X$ is the locally finite topology $e^{\tau}$.

In view of the relationship between UC spaces and Atsuji spaces, the compactness of $X^{\prime}$ is an important property. We will now show that first countable hyperspaces can arise only when $X$ is at least countably compact.

THEOREM 2.7. Let $(X, \tau)$ be normal. If $\left(2^{X}, e^{\tau}\right)$ is first countable, then $X^{\prime}$ is countably compact.

ProOF. Suppose $X^{\prime}$ is not countably compact, and let $E=\left\{x_{n}: n<\omega\right\}$ be a countably infinite subset of $X^{\prime}$ having no limit point in $X$. As $X$ is normal, the closed set $E$ is $C$-embedded in $X$, and thus, we can find a discrete collection $\left\{G_{n}: n<\omega\right\}$ of open sets in $X$ such that $x_{n} \in G_{n}$ for each $n$. Using the first countability hypothesis on $e^{\tau}$, and Lemma 1.1, we construct a countable base $\left\{\mathscr{A}_{n}^{\wedge}: n<\omega\right\}$ at $E \in 2^{X}$, such that for each $n<\omega, \mathscr{A}_{n}=\left\{P_{n 1}, P_{n 2}, \ldots\right\}$ is a discrete collection of open subsets of $X, x_{m} \in P_{n m}$ for each $m<\omega$, and $\mathscr{A}_{n+1} \subseteq \mathscr{A}_{n}$. As each $x \in E$ is a limit point of $X$, each of the sets $P_{m n}$ is infinite. Choosing any $y_{n} \in P_{n n}-\left\{x_{n}\right\}$ and setting $Q_{n}=P_{n n}-\left\{y_{n}\right\}$, we obtain an open set $\left\{Q_{n}: n<\omega\right\}$ containing $E \in 2^{X}$ such that no $\mathscr{A}_{n}^{\widehat{\imath}}$ is contained in this set. This is contrary to the fact that $\left\{\mathscr{A}_{n} \hat{:} n<\omega\right\}$ is, by our construction, a countable base at the point $E$. Thus, we conclude that under the given hypotheses, $X^{\prime}$ must indeed be countably compact. The proof of the theorem is now complete.

COROllaRY 2.8. Let $(X, \tau)$ be a paracompact $T_{2}$ space. If $\left(2^{X}, e^{\tau}\right)$ is first countable, $X^{\prime}$ must be compact.

Specializing the preceding corollary to the class of self-dense, paracompact, Hausdorff spaces, we find that only very special spaces belonging to this class can ensure the first countability of $e^{\tau}$. The precise result is as follows:

COROllary 2.9. Let $(X, \tau)$ be a dense in itself, paracompact, Hausdorff space. The following conditions are equivalent:

(a) $\left(2^{X}, e^{\tau}\right)$ is first countable.

(b) $\left(2^{X}, e^{\tau}\right)$ is compact and first countable.

(c) $(X, \tau)$ is compact and first countable.

Proof. That $(\mathrm{a}) \Rightarrow(\mathrm{c})$ follows from Corollary 2.8 and the fact that $X$ is embedded in $2^{X}$. To see that $(\mathrm{c}) \Rightarrow(\mathrm{b})$, note that, by $(\mathrm{c}), 2^{\tau}=e^{\tau}$, and recall that under (c), $2^{\tau}$ is compact and first countable $[\mathbf{1 1}]$. That $(\mathrm{b}) \Rightarrow(\mathrm{a})$ is clear.

A well-known result (cf. Theorem 15.3 of [12]) about hyperspace topologies states that if $\mathscr{U}$ and $\mathscr{V}$ are two uniformities on a set, $\mathscr{U}$ totally bounded, and if $\left|2^{\mathscr{U}}\right| \subseteq\left|2^{\mathscr{V}}\right|$, then $\mathscr{U} \subseteq \mathscr{V}$. This combined with Theorem 2.7 leads to the following characterizations of UC spaces given in [4].

COROLlaRY 2.10. Let $(X, \tau)$ be a metrizable space. The following conditions are equivalent:

(a) $e^{\tau}$ is metrizable.

(b) $e^{\tau}$ is first countable.

(c) $X^{\prime}$ is compact. 
(d) There is a compatible metric $d$ that makes $X$ a UC space.

(e) There is a compatible metric $d$ for which $e^{\tau}=\left|2^{\mathscr{U}(d)}\right|$.

(f) There is a compatible metric $d$ for which $2^{\tau} \subseteq\left|2^{\mathscr{U}(d)}\right|$.

The authors would like to thank the referee for many valuable suggestions which greatly helped to improve the notation.

\section{BIBLIOGRAPHY}

1. M. Atsuji, Uniform continuity of continuous functions of metric spaces, Pacific J. Math. 8 (1958), 11-16.

2. G. Beer, Metric spaces on which continuous functions are uniformly continuous and Hausdorff distance, Proc. Amer. Math. Soc. 95 (1985), 653-658.

3. __ More about metric spaces on which continuous functions are uniformly continuous, Bull. Austral. Math. Soc. 33 (1986), 397-406.

4. G. Beer, C. J. Himmelberg, K. Prikry, and F. Van Vleck, The locally finite topology on $2^{X}$, Proc. Amer. Math. Soc. 101 (1987), 168-172.

5. R. Engelking, General topology, Polish Sci. Publ., Warsaw, 1977.

6. L. Gillman and M. Jerison, Rings of continuous functions, Springer-Verlag, New York, 1976.

7. C. J. Himmelberg, K. Prikry, and F. Van Vleck, The Hausdorff metric and measurable selections, Topology Appl. 20 (1985), 121-133.

8. J. L. Kelley, General topology, D. Van Nostrand, Princeton, N. J., 1955.

9. J. Kerstan, Zur charakterisierung der pseudokompakten, Math. Nachr. 16 (1957), 289-293.

10. M. Marjanovic, Topologies on collections of closed sets, Publ. Inst. Math. (Beograd) (N.S.) 6 (1966), 125-130.

11. E. Michael, Topologies on spaces of subsets, Trans. Amer. Math. Soc. 71 (1951), 152-182.

12. S. A. Naimpally and B. D. Warrack, Proximity spáces, Cambridge Univ. Press, Cambridge, 1970.

13. J. Rainwater, Spaces whose finest uniformity is metric, Pacific J. Math. 9 (1959), 567-570.

14. W. Waterhouse, On UC spaces, Amer. Math. Monthly 72 (1965), 634-635.

15. S. Willard, General topology, Addison-Wesley, Reading, Mass., 1970.

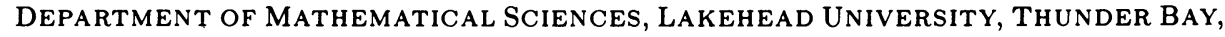
ONTARIO P7B 5E1, CANADA

DEPARTMENT OF MATHEMATICAL SCIENCES, BUTLER UNIVERSity, INDiANAPOLIS, INDIANA 46208 